\title{
Magnetic resonance imaging of the right ventricle in pediatric pulmonary arterial hypertension
}

\author{
Shannon Blalock ${ }^{1}$, Frandics Chan ${ }^{4}$, David Rosenthal ${ }^{2}$, Michelle Ogawa', Dawn Maxey', Jeffrey Feinstein ${ }^{2,3}$ \\ ${ }^{1}$ Department of Pediatrics, University of Texas Southwestern, Dallas, Texas, USA; ${ }^{2}$ Department of Pediatrics; ${ }^{3}$ Bioengineering and \\ ${ }^{4}$ Radiology, Stanford University, Palo Alto, California, USA
}

\begin{abstract}
Pulmonary arterial hypertension (PAH) causes changes in the right ventricle (RV), affecting RV size and function, ultimately leading to death. These changes have been evaluated by cardiac MRI (CMR) in adults with PAH, but not in children. Using CMR in pediatric patients with PAH, we examined how RV size and function (1) compare to normal data, (2) change over time, and (3) compare to similar studies in the adult population. Data from two institutions were retrospectively reviewed. Subjects with PAH and a CMR were included. Baseline CMR variables (right and left ventricular end-diastolic and end-systolic volumes indexed for body surface area, and calculated stroke volume and ejection fraction) were compared to normative data and follow-up CMR data. Twenty-six subjects (15 female), age 2-16 (mean 11) years, with idiopathic PAH were included. All patients were on PAH medication, and $65 \%$ on prostacyclin therapy. The baseline 6-Minute Walk Distance (6MWD; $481 \pm 137$ ) was normal. RV volumes and ejection fraction were markedly abnormal compared to normal data $(P<0.001)$. Follow-up CMRs were analyzed in 15 patients. $\mathrm{RV}$ volumes and function and LV stroke volume showed no significant change over one year. Our pediatric patients with PAH have markedly abnormal right ventricles by CMR but have normal walk distances. The lack of change in CMR parameters over one year may represent a stable cohort and is different than similar studies in adults.
\end{abstract}

Key Words: cardiac magnetic resonance, pediatrics, pulmonary hypertension, right ventricle

Idiopathic pulmonary arterial hypertension (IPAH) is a progressive disease which, if left untreated, is ultimately fatal. Elevated pulmonary artery pressures increase the workload on the right ventricle (RV) which undergoes a series of changes, initially thickening as a compensatory mechanism but then eventually dilating and failing while compressing the left ventricle and inhibiting cardiac output. ${ }^{[1]}$ Barst et al ${ }^{[2]}$ suggest that although children have evidence of more severe disease at diagnosis, based on hemodynamic data by catheterization, they tolerate this increased workload on the RV better than adults. Despite early evidence of worse survival in children compared to adults, the introduction of epoprostenol and other targeted therapies into the pharmacological arsenal has increased the survival in children to a level similar to that in adults - an estimated $86 \%$ at four years by one recent US retrospective study, and $72 \%$ at five years from the UK

Address correspondence to:

Dr. Shannon Blalock

1935 Medical District Drive

Division of Cardiology

Dallas, TX 75235, USA

Email: Shannon.blalock@childrens.com pulmonary hypertension service ${ }^{[3-5]}$ Despite this increased survival, disease progression is still a concern as evidenced by the $25 \%$ long-term mortality ${ }^{[5]}$ Given the correlation of right ventricular health with prognosis, direct evaluation of the right ventricular structure and function may be the best method to observe progression of the disease. ${ }^{[6,7]}$ Evaluation of the ventricular morphological and functional changes in the adult PAH population is increasingly performed by cardiac magnetic resonance (CMR) imaging for its accuracy, objectivity, and low inter-study variability. ${ }^{[8]}$ Recent studies have shown the prognostic significance of CMR parameters in adults with IPAH ${ }^{[8-11]}$ To date, there is very little information regarding assessment of the ventricular structure or function by CMR in pediatric PAH.

\begin{tabular}{|l|l|}
\hline \multicolumn{2}{|c|}{ Access this article online } \\
\hline Quick Response Code: & Website: www.pulmonarycirculation.org \\
\hline & DOI: 10.4103/2045-8932.114763 \\
\cline { 2 - 3 } & $\begin{array}{l}\text { How to cite this article: Blalock S, Chan F, } \\
\text { Rosenthal D, Ogawa M, Maxey D, Feinstein } \\
\text { J. Magnetic resonance imaging of the right } \\
\text { ventricle in pediatric pulmonary arterial } \\
\text { hypertension. Pulm Circ 2013;3:350-5. }\end{array}$ \\
\hline
\end{tabular}


Using cardiac magnetic resonance imaging, we examined how the right ventricle in pediatric patients with $\mathrm{PAH}$ compares to historical normative data, and whether these measures of right ventricular size and function change over time and have similar findings to studies in adults.

\section{MATERIALS AND METHODS}

\section{Subjects}

Pediatric patients followed in the pulmonary hypertension clinic from two tertiary-care centers, Children's Medical Center Dallas (CMC) in Dallas, Texas and Lucile Packard Children's Hospital (LPCH) in Palo Alto, California were screened. Patients with a diagnosis of IPAH, confirmed by cardiac catheterization, who also had a CMR performed as routine care between September 2005 and June 2011 were included in the study. Demographic information, $6 \mathrm{MWD}$, cardiac catheterization, and laboratory data performed at the time of the CMRs were retrospectively collected. Patients with secondary causes of PAH, including chronic lung disease, connective tissue disease, and congenital heart disease, were excluded. This study complies with the Declaration of Helsinki, and an institutional review board approved the study with a waiver of informed consent. The historical controls used for CMR normal values were 70 children age 8-20 years, gender-matched to our patients. ${ }^{[12]}$

\section{MR imaging protocol}

Imaging was performed using a Philips 1.5 Tesla MRI scanner (Philips Medical Systems, The Netherlands) at CMC and a GE SignaTwinSpeed 1.5 Tesla MRI scanner (General Electric Medical Systems, Milwaukee, Wisc., USA) at LPCH. Patients were placed in supine position within a phase-array cardiac coil, with respiratory monitor and ECG-leads attached, and intravenous access established. Patients under the age of eight underwent general anesthesia with breath-holds for the CMR. After localizer sequences, multi-slice, breath-held, ECG-gated balanced steady-state free precession cine images were obtained in the short-axis plane. Scan parameters were $\mathrm{TE}=1 \sim 2 \mathrm{~ms}, \mathrm{TR}=3 \sim 4 \mathrm{~ms}$, flip angle $=45-60$ degree, field-of-view $=28-36 \mathrm{~cm}$, slice thickness $=6 \sim 8 \mathrm{~mm}$, no gap. Depending on the heart rate, the number of scan lines per R-R interval was $8 \sim 14$, yielding a temporal resolution about $40 \mathrm{~ms}$ and a breath-hold time about 20 seconds. Twenty to 30 cardiac phases were reconstructed. Delayed enhancement imaging was performed after the intravenous injection of $0.4 \mathrm{mmol} / \mathrm{kg}$ of gadolinium contrast agent (Magnevist, Bayer HealthCare, Wayne, N.J., USA) and 6-10-minute delay at LPCH. Breath-held, ECGgated, single phase, inversion-recovery gradient-recalled echo sequence was performed in the short-axis plane. The inversion time, typically $160-330 \mathrm{~ms}$, was adjusted to suppress normal myocardial signal.

\section{Image analysis}

Offline analysis of ventricular volumes, mass, and calculated ejection fraction were performed on a ViewForum workstation (Philips Medical Systems) at CMC and a GE Advantage workstation (General Electric Medical Systems) at LPCH. End-systolic and end-diastolic frames were identified as the smallest and largest volumes, respectively, in separate short-axis views of both ventricles. The endocardial borders were traced to determine the area and the software computed volumes by multiplying the area by slice thickness. The ventricular ejection fraction (EF) was calculated using the standard equation $\mathrm{EF}=(\mathrm{EDV}-\mathrm{ESV}) / \mathrm{EDV} \times 100 \%$. As no patient had more than mild mitral regurgitation, the left ventricular stroke volume (LVEDVi-LVESVi) was used as a surrogate for cardiac output. ${ }^{[13]}$ The right ventricular mass was calculated from the end-diastolic endocardial and epicardial tracing of the right ventricle in the short-axis plane. The inter-ventricular septum was not included in the right ventricular mass in accordance with the method of Hudsmith. ${ }^{[14]}$ CMR variables were indexed to body surface area (meters-squared).

\section{Cardiac catheterization}

Right heart catheterization was performed using a balloon-tipped catheter in patients undergoing general anesthesia at CMC and under deep sedation at LPCH. Mean right atrial pressure, right ventricular systolic and diastolic pressures, systolic pulmonary artery pressures, pulmonary capillary wedge pressure, systemic venous saturation, and arterial saturation based on pulse oximetry was obtained in each patient. The cardiac index was calculated according to the Fick principle as previously published. ${ }^{[15]}$ The baseline cardiac catheterization data reported were baseline measurements on minimal oxygen.

\section{Six-minute walk distance}

The 6-Minute Walk Distance test was administered by an experienced staff member and was performed indoors on a flat surface according to standard protocol. ${ }^{[16]}$

\section{Statistical analysis}

All data are expressed as means with standard deviation, unless otherwise stated. The indexed CMR mean values and baseline 6MWD in study subjects were compared with published normative data using an unpaired $t$-test. For comparison between baseline and follow-up CMR variables in the same patients, the nonparametric Wilcoxon signed rank test was used. Improvement or worsening was defined as a greater than $5 \%$ change between the baseline and follow-up study. The correlation between baseline CMR variables, catheterization data and 6MWD were determined by nonparametric (Spearman) correlation. Categorical echocardiographic data was compared to CMR data by creating categories of RV dysfunction based on pediatric normative data by $\mathrm{CMR}^{\left[{ }^{[2]}\right.}$ The RVEF by CMR categories were 
normal (55-70\%), mild dysfunction (45-54\%), moderate dysfunction (35-44\%), and severe dysfunction $(<35 \%)$. RV size by CMR was deemed "enlarged" if greater than 2 standard deviations from the mean as expressed by the normative data based on gender ${ }^{[12]} \mathrm{A}$ criterion of significance (alpha) of 0.05 was used (SPSS 15.0, Chicago, Ill., USA).

\section{RESULTS}

Twenty-six subjects, mean age 10.8 years (range 2-16 years) with IPAH were included. No patient had an adverse event during or immediately following the CMR. Patient demographics, catheterization, and walk data at the time of the baseline CMR are shown in Table 1. Seventeen patients (65\%) were prescribed intravenous or subcutaneous prostacyclin. Seventeen patients had a cardiac catheterization performed within 90 days of their baseline CMR (mean 3 days \pm 40 days). Baseline catheterization data showed abnormal elevations in mean right atrial pressure, systolic pulmonary artery pressure, pulmonary vascular resistance index, and pulmonary to systemic vascular resistance ratio (PVRi/SVRi). Twenty-two patients had a baseline 6MWD performed within 90 days of the CMR (mean $20 \pm 45$ days). The mean distance, $481 \pm 137$ meters, was the same as normal pediatric values for 4- to 11-yearolds $(470 \pm 59, P=0.15) \cdot{ }^{[17]}$ Three patients could not perform a walk test due to young age and one patient had a 6MWD greater than 90 days from the date of the CMR.

Baseline CMR data in the study subjects showed markedly abnormal right and left ventricular volumes and function compared to normal data (Table 2). ${ }^{[12]}$ Specifically, there was evidence of elevated right ventricular mass and end-diastolic volumes with decreased right ventricular ejection fraction and left ventricular stroke volume in both sexes.

Baseline CMR data was also evaluated for evidence of myocardial delayed enhancement. At one institution, eleven patients had myocardial delayed enhancement performed during the baseline CMR study. All eleven patients had evidence of delayed enhancement at the insertion points of the right ventricle to the interventricular septum $(80 \%$ anterior, 70\% inferior), consistent with previous studies in the adult population corresponding to areas of abnormal myocardium. ${ }^{[18,19]}$

To evaluate how the ventricles change over time, we selected patients with two or more CMR scans within our study period. Seventeen patients had repeat scans. One patient was excluded due to poor image quality and inability to perform offline analysis. Another patient was excluded due to the interval placement of an atrial stent with significant improvement in the ventricular volumes and function. Fifteen patients were included in the analyses, with a mean interval of $1.25 \pm 0.4$ years between the studies. The baseline versus follow-up clinical data is given in the appendix. The right ventricular end-diastolic volumes and ejection fraction did not change significantly compared to baseline studies $(P=0.1, P=0.5$, respectively; Figures. $1 \mathrm{~A}$ and $\mathrm{B})$. The left ventricular stroke volume increased from baseline (median $40 \mathrm{ml} / \mathrm{m}^{2}$ ) to follow-up (median $46 \mathrm{ml} / \mathrm{m}^{2}, P=0.007$; Fig. 1C). No patient had significant improvement in both volume and function. Only three patients (appendix subjects \#7, 12, and 13) had deterioration in both volume and function. Significant change was determined by a greater than $5 \%$ change. Of the three, two had medication changes. One had the addition of sildenafil and the other had a change from bosentan to ambrisentan and iloprost to treprostinil. None of these patients died or went to be listed for transplantation. We have one patient currently listed for transplantation (appendix subject \#15) and two patients who received a transplant, one of whom later died. The patient who died had a baseline MRI which showed an RVEF of $22 \%$, RVEDVi of $213 \mathrm{ml} / \mathrm{m}^{2}$, and $\mathrm{LV}$ stroke volume of $42 \mathrm{ml} / \mathrm{m}^{2}$.

\section{DISCUSSION}

Our study is exploratory and the first to characterize the right ventricle by CMR in pediatric patients with $\mathrm{PAH}$. We have shown CMR to be a feasible method of directly assessing the structural and functional characteristics of the ventricles.

At baseline, our pediatric patients have markedly abnormal RV size and function by CMR compared to normative data. In addition, they have evidence of abnormal myocardium by delayed enhancement at the RV insertion points, which is seen in adults with PAH and other diseases with increased RV afterload. ${ }^{[18,20]}$ These abnormal morphological and functional findings by CMR are consistent with the abnormal baseline hemodynamics in our study population, a large portion of whom have severe disease. However, these children tolerate the abnormal ventricular changes without detriment to their exercise capacity, as evidenced by the normal baseline 6 MWD. This is unlike adults who have abnormal 6 MWD along with abnormal CMR findings, despite treatment. ${ }^{[21]}$

Previous studies have suggested that pediatric patients with PAH appear to handle increased workload on the right ventricle better than adults, but the mechanism of this adaptation is unknown ${ }^{[2]}$ Again, unlike adults, in our study there is no significant change in CMR RV variables from baseline to follow-up at an average of 1.2 years in treated patients. Recent adult studies have shown a significant deterioration in RV and LV parameters over 1 year in a majority of patients. ${ }^{[8,9]}$ Perhaps our patients represent a stable cohort who has markedly abnormal baseline RV parameters but do not change over time. 


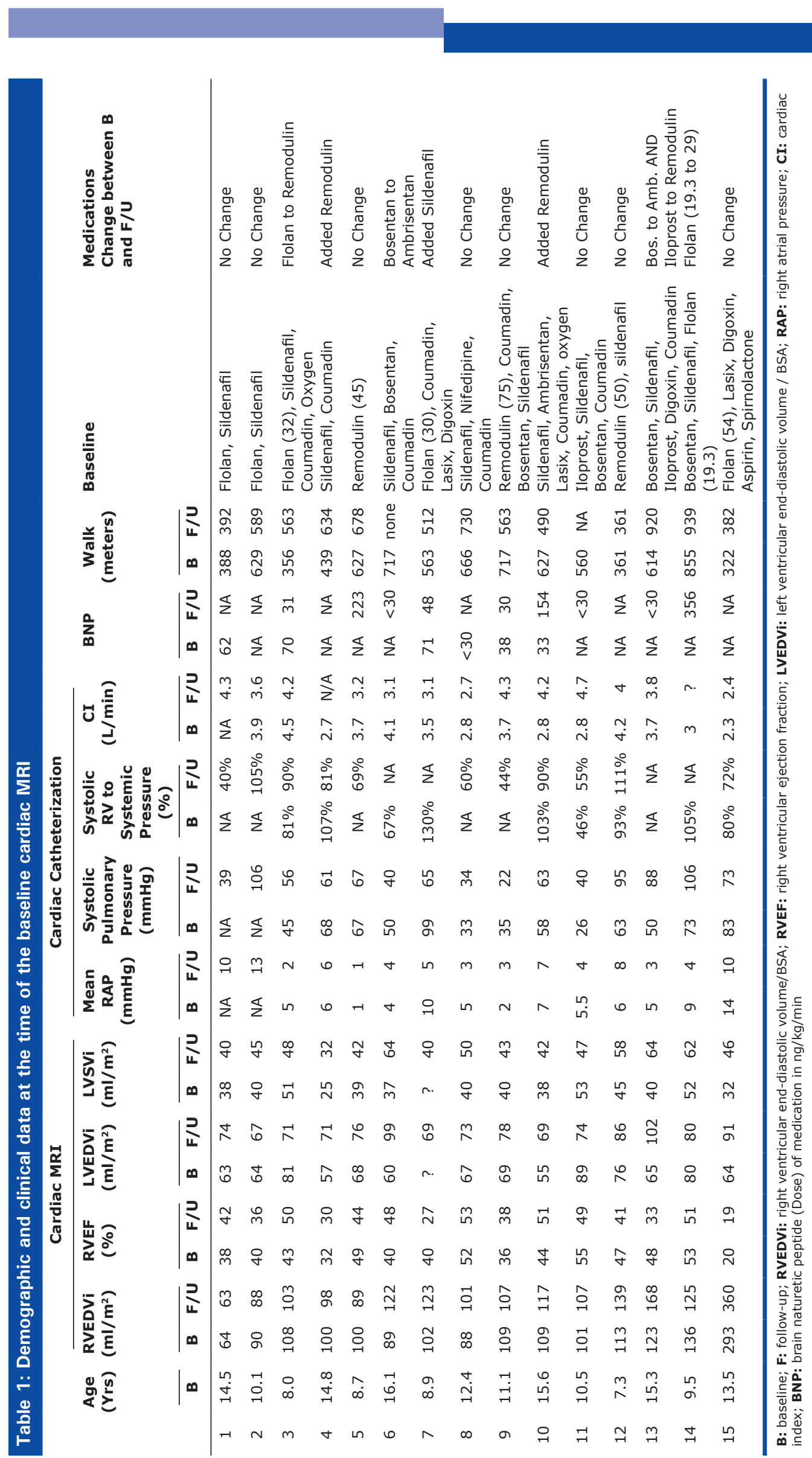


One theory as to why pediatric patients tolerate the increased workload could be the preservation of an adequate stroke volume (SV), which has been suggested to be a direct assessment of the RV response to load, without the additional confounding factor of increased heart rate (as in the case of cardiac output). ${ }^{[9]}$ Only two of our patients had an SV $<25 \mathrm{ml} / \mathrm{m}^{2}$, which was the adult threshold for increased mortality in the study by Van Wolferen et al. ${ }^{[8]}$ It appears that despite the deleterious structural changes of both ventricles, the stroke volume is sufficient, which may result in the superior exercise capacity in our patients.

Interestingly, the other two CMR variables which showed prognostic significance in the univariate, multivariate, and Kaplan-Meier survival analysis of the Van Wolferen study (LVEDVi and RVEDVi) did not show the same prognostic significance in children. For example, their study found adult patients with an RV end-diastolic volume $\geq 84 \mathrm{ml} / \mathrm{m}^{2}$ had a $50 \%$ survival at 1 year. At baseline, all but two of our pediatric patients $(n=24)$ had an RVEDV $\geq 84 \mathrm{~m} / \mathrm{m}^{2}$, which would predict at least 12 deaths. We had only one pediatric death. This adult prognostic indicator does not appear to be transferrable to the pediatric population. However, we recognize the small number of patients in our study as a limitation compared to the adult studies.

Other adult endpoints such as functional class and quality of life are difficult to assess in young children. ${ }^{[22]}$ Due to the lack of overt symptoms in children and their ability to maintain exercise capacity despite having evidence of severe disease, we continue to look for the gold standard in which subtle deterioration can be detected before it is clinically evident. Although adult CMR indicators of survival are not transferable to the pediatric population, perhaps, with further studies, we will determine which parameters, or change in parameters, will be an accurate prognostic indicator in our pediatric population. In our limited and small study, we suggest that the lack of change in parameters, particularly LV stroke volume, represents a favorable prognostic finding. Longer followup and additional, larger studies are needed to test this hypothesis.

\section{Limitations}

This study is limited by the small sample size, variability between institutions, lack of standardized collection of laboratory data, and the lack of a defined gold standard for evaluation of these pediatric patients with $\mathrm{PAH}$. Newer techniques by CMR may be helpful in evaluating these patients by CMR, but were not available at the commencement of this study. In addition, the majority of our study subjects did not have significant CMR changes over one year which may limit the usefulness of frequent MRI studies in this patient population.

In conclusion, despite normal 6MWD in children with PAH, markedly abnormal right and left ventricles are assessed by CMR parameters. These MRI parameters do not significantly

Table 2. Baseline Cardiac MRI data compared to normal patients

\begin{tabular}{|c|c|c|c|c|c|c|}
\hline & \multicolumn{3}{|c|}{ Males } & \multicolumn{3}{|c|}{ Females } \\
\hline & Normal $n=35$ & PAH $n=11 *$ & & Normal $n=35$ & PAH $n=15 *$ & \\
\hline RVEDVi (ml/m2) & $82.9 \pm 12.6$ & $128 \pm 59$ & $P<0.001$ & $78.3 \pm 9.7$ & $113 \pm 38$ & $P<0.001$ \\
\hline RVEF (\%) & $62.3 \pm 4.3$ & $39 \pm 15$ & $P<0.001$ & $62.6 \pm 3.6$ & $43 \pm 13$ & $P<0.001$ \\
\hline RV mass $(\mathrm{g} / \mathrm{m} 2)$ & $18.3 \pm 5.5$ & $43 \pm 37$ & $P<0.001$ & $17.3 \pm 4.7$ & $35 \pm 17$ & $P<0.001$ \\
\hline LVEDVi $(\mathrm{ml} / \mathrm{m} 2)$ & $81.9 \pm 12.9$ & $67 \pm 12$ & $P=0.002$ & $78.7 \pm 10.7$ & $67 \pm 15$ & $P=0.002$ \\
\hline LVEF (\%) & $65.7 \pm 4.9$ & $58 \pm 8$ & $P<0.001$ & $63.2 \pm 6.3$ & $62 \pm 10$ & $P=0.46$ \\
\hline LVSVi (ml/m2) & $54.6 \pm 8.2$ & $39 \pm 10$ & $P<0.001$ & $49.7 \pm 8.3$ & $41 \pm 10$ & $P=.0002$ \\
\hline
\end{tabular}

RVEDVi: right ventricular end-diastolic volume indexed for body surface area; RVEF: right ventricular ejection fraction; RV mass: right ventricular mass; LVEDVi: left ventricular end-diastolic volume indexed for body surface area; LVEF: left ventricular ejection fraction; LVSVi: left ventricular stroke volume (LVEDV-LVESV) indexed for body surface area; PAH: pulmonary arterial hypertension. *For RV mass $\mathrm{n}=6$ males, 7 females
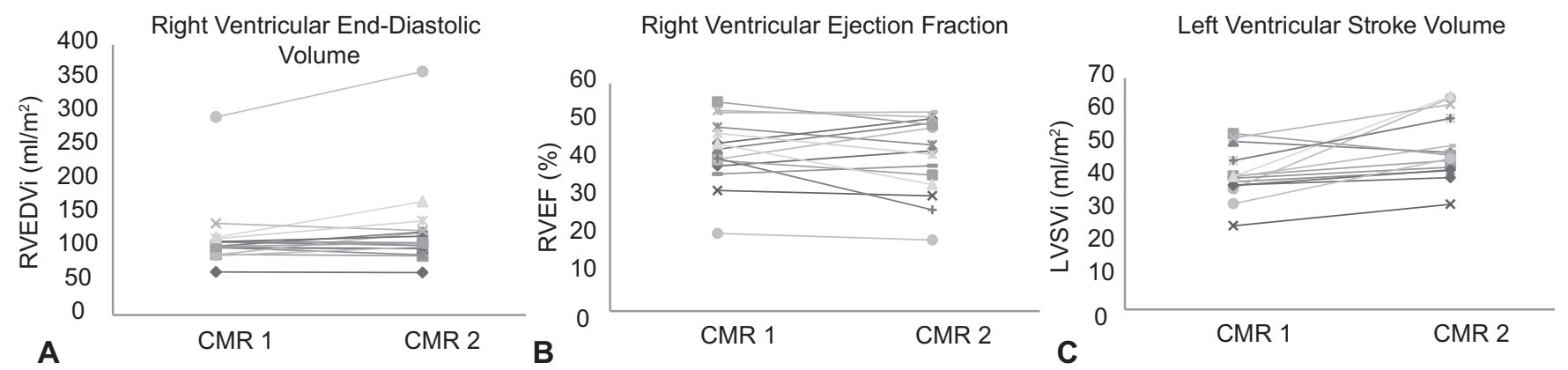

Figure 1: (A) Change in right ventricular end-diastolic volume, (B) ejection fraction, and (C) left ventricular stroke volume from baseline to follow-up; mean interval of $1.2 \pm 0.4$ years. 
change in 1 year. CMR markers in the adult population do not seem transferable to the pediatric population.

\section{REFERENCES}

1. Chin KM, Kim NH, Rubin LJ. The right ventricle in pulmonary hypertension. Coron Artery Dis 2005;16:13-8.

2. Barst RJ, Ertel SI, Beghetti M, Ivy DD. Pulmonary arterial hypertension: A comparison between children and adults. Eur Respir J 2011;37:665-77.

3. D'Alonzo GE, Barst RJ, Ayres SM, Bergofsky EH, Brundage BH, Detre KM, et al. Survival in patients with primary pulmonary hypertension. Results from a national prospective registry. Ann Intern Med 1991;115:343-9.

4. Haworth SG, Hislop AA. Treatment and survival in children with pulmonary arterial hypertension: The UK Pulmonary Hypertension Service for Children 2001-2006. Heart 2009;95:312-7.

5. Ivy DD, Rosenzweig EB, Lemarie JC, Brand M, Rosenberg D, Barst RJ. Longterm outcomes in children with pulmonary arterial hypertension treated with bosentan in real-world clinical settings. Am J Cardiol 2010;106:1332-8.

6. Badano LP, Ginghina C, Easaw J, Murraru D, Grillo MT, Lancellotti P, et al. Right ventricle in pulmonary arterial hypertension: Haemodynamics, structural changes, imaging, and proposal of a study protocol aimed to assess remodelling and treatment effects. Eur J Echocardiogr 2010;11:27-37.

7. Badesch DB, Champion HC, Sanchez MA, Hoeper MM, Lyod JE, Manes A, et al. Diagnosis and assessment of pulmonary arterial hypertension. J Am Coll Cardiol 2009;54:S55-66.

8. van Wolferen SA, Marcus JT, Boonstra A, Marques KM, Bronzwaer JG, Spreeuwenberg MD, et al. Prognostic value of right ventricular mass, volume, and function in idiopathic pulmonary arterial hypertension. Eur Heart J 2007;28:1250-7.

9. van Wolferen SA, van de Veerdonk MC, Mauritz GJ, Jacobs W, Marcus JT, Marques KM, et al. Clinical significant change of stroke volume in pulmonary hypertension. Chest 2010;139:1003-9.

10. Van Praagh S, Geva T, Lock JE, Nido PJ, Vance MS, Van Praagh R. Biatrial or left atrial drainage of the right superior vena cava: Anatomic, morphogenetic, and surgical considerations: Report of three new cases and literature review. Pediatr Cardiol 2003;24:350-63.

11. Fakhri AA, Hughes-Doichey RA, Beiderman RW, Murali S. Imaging in the evaluation of pulmonary artery hemodynamics and right ventricular structure and function. Heart Fail Clin 2012;8:353-72.

12. Sarikouch S, Peters B, Gutberlet M, Leismann B, Kelter-Kloepping A, Koerperich $\mathrm{H}$, et al. Sex-specific pediatric percentiles for ventricular size and mass as reference values for cardiac MRI: Assessment by steady-state freeprecession and phase-contrast MRI flow. Circ Cardiovasc Imaging 2010;3:65-76.

13. Mauritz GJ, Marcus JT, Boonstra A, Postmus PE, Westerhof N Vonk-Noordegraaf A. Non-invasive stroke volume assessment in patients with pulmonary arterial hypertension: Left-sided data mandatory. J Cardiovasc Magn Reson 2008;10:51.

14. Hudsmith LE, Petersen SE, Francis JM, Robson MD, Neubauer S. Normal human left and right ventricular and left atrial dimensions using steady state free precession magnetic resonance imaging. J Cardiovasc Magn Reson 2005;7:775-82.

15. Wilmore J, Hamra M. Physiology of Exercise in Children. In: The Science and Practice of Pediatric Cardiology. 2nd ed, Vol 1. Baltimore, Maryland: Williams and Wilkins; 1998. p. 447.

16. ATS Committee on Proficiency Standards for Clinical Pulmonary Function Laboratories. ATS statement: Guidelines for the six-minute walk test. Am J Respir Crit Care Med 2002;166:111-7.

17. Lammers AE, Hislop AA, Flynn Y, Haworth SG. The 6-minute walk test Normal values for children of 4-11 years of age. Arch Dis Child 2008;93:464-8.

18. Blyth KG, Groenning BA, Martin TN, Foster JE, Mark PB, Dargie HJ, et al Contrast enhanced-cardiovascular magnetic resonance imaging in patients with pulmonary hypertension. Eur Heart J 2005;26:1993-9.

19. McCann GP, Beek AM, Vonk-Noordegraaf A, van Rossum AC. Delayed contrast-enhanced magnetic resonance imaging in pulmonary arterial hypertension. Circulation 2005;112:e268.

20. McCann GP, Gan CT, Beek AM, Niessen HW, Vonk Noordegraaf A, van Rossum AC. Extent of MRI delayed enhancement of myocardial mass is related to right ventricular dysfunction in pulmonary artery hypertension. AJR Am J Roentgenol 2007;188:349-55.

21. Chin KM, Kingman M, de Lemos JA, Warner JJ, Reimond S, Peshock R, et al. Changes in right ventricular structure and function assessed using cardiac magnetic resonance imaging in bosentan-treated patients with pulmonary arterial hypertension. Am J Cardiol 2008;101:1669-72.

22. Haworth SG, Beghetti M. Assessment of endpoints in the pediatric population: Congenital heart disease and idiopathic pulmonary arterial hypertension. Curr Opin Pulm Med 2010;16 Suppl 1:S35-41.

Source of Support: None, Conflict of Interest: None declared. 